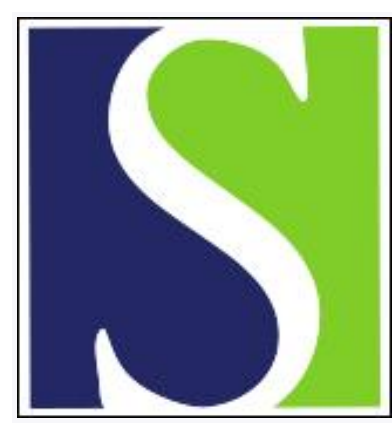

Scand J Work Environ Health 1987;13(4):275-278

https://doi.org/10.5271/sjweh.2038

Issue date: Aug 1987

The Stockholm Workshop scale for the classification of cold-induced Raynaud's phenomenon in the hand-arm vibration syndrome (revision of the Taylor-Pelmear scale). by Gemne G, Pyykko I, Taylor W, Pelmear PL

Affiliation: National Institute of Occupational Health, Unit of Occupational Medicine, Solna, Sweden.

This article in PubMed: www.ncbi.nlm.nih.gov/pubmed/3433028

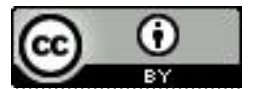




\title{
The Stockholm Workshop scale for the classification of cold-induced Raynaud's phenomenon in the hand-arm vibration syndrome (revision of the Taylor-Pelmear scale)
}

\author{
by Gösta Gemne, MD,PhD, ${ }^{1}$ IImari Pyykkö, MD,PhD, ${ }^{2}$ William Taylor, DSc,MD,FRCP,FFOM, ${ }^{3}$ \\ Peter L Pelmear, MD,FFOM,CCBOM ${ }^{4}$
}

\begin{abstract}
GEMNE G, PYYKKÖ I, TAYLOR W, PELMEAR PL. The Stockholm Workshop scale for the classification of cold-induced Raynaud's phenomenon in the hand-arm vibration syndrome (revision of the TaylorPelmear scale). Scand J Work Environ Health 13 (1987) 275-278. On the basis of experience accumulated over the past few years, a revision has been made in the currently used Taylor-Pelmear scale for the staging of Raynaud's phenomenon in persons exposed to vibration from hand-held tools, while retaining as much as possible of the well-established advantages of the scale for research and its proved usefulness for clinical and medicolegal purposes. The $0_{\mathrm{T}}$ and $0_{\mathrm{N}}$ stages of symptoms have been omitted, together with the parallel disability scale. A separate staging for neurological disorders connected with the syndrome was proposed and accepted at the workshop "Symptomatology and Diagnostic Methods in the Hand-Arm Vibration Syndrome," held in Stockholm in 1986. The criteria descriptions have been changed so as to minimize their reliance on seasonal factors. The new staging system - a stage 0 and four stages (1-4) with attacks of cold-induced Raynaud's phenomenon - clearly defines the differences in the descriptions of the stage criteria in order to improve their clinical usefulness. A numerical scoring based on the extent and distribution of finger blanching was not, however, introduced, whereas a score based on the number of affected fingers on each hand was proposed, considered, and accepted.
\end{abstract}

Key terms: hand-held vibrating tools, Raynaud's phenomenon, vibration-induced white fingers, VWF.

An assessment of the subjective symptoms of vascular and neurological disturbances manifested in the handarm vibration syndrome is essential to clinical work and research. At the Stockholm workshop entitled "Symptomatology and Diagnostic Methods in the Hand-Arm Vibration Syndrome" a session was devoted to a discussion of this question. The startingpoint was a contribution by Taylor, who gave a comprehensive survey of the background of the currently used Taylor-Pelmear scale $(10,12)$ and the special considerations required to carry out a correct staging. It also dealt with the usefulness of the scale for research, for practical clinical work, and for medicolegal purposes.

\section{Motives for revision}

It became clear from the discussions that this scale should be revised. The main reason commonly ex-

\footnotetext{
1 National Institute of Occupational Health, Unit of Occupational Medicine, Solna, Sweden.

2 Department of Physiology, Institute of Occupational Health, Helsinki, Finland.

3 Department of Community Medicine, University of Dundee, Dundee, Scotland.

4 Occupational Health Branch, Ontario Ministry of Labour, Toronto, Ontario, Canada.
}

Reprint requests to: Dr G Gemne, National Institute of Occupational Health, Unit of Occupational Medicine, S-171 84 Solna, Sweden. pressed was that difficulties had been experienced in the staging of research subjects and patients.

The scale revision needed to include the following:

1. A general amendment of the Taylor-Pelmear scale to single out the vascular component of the hand-arm vibration syndrome. This revision would require accentuating the criteria differences between the stages, eliminating seasonal criteria from the staging, and indicating that the scale should be used exclusively for the staging of cold-induced Raynaud's phenomenon.

2. The separation of the scale components based on neurological symptoms from those purely concerning circulatory disturbances. This revision would be necessary to eliminate the ambiguities arising from the intermingling of variables denoting white fingers with those of neurological symptoms (the " $0_{\mathrm{N}}$ " and " $0_{\mathrm{T}}$ " stages, and other references to neurological symptoms like "tingling" and "numbness"). A scale entirely dedicated to disorders of peripheral nerve function would be required.

3. The elimination of the disability scale. That the disability stages parallel the vascular stages has been questioned. This part of the scale is subjective, and responses by patients vary widely (to an unknown extent) due to differences in, for instance, socioeconomic factors. 


\section{Separation of vascular and neurological components}

There are no epidemiologic data on the relationship between the various vascular symptoms and the development or occurrence of the paresthesias covered by the $0_{\mathrm{N}}$ and $0_{\mathrm{T}}$ stages of the Taylor-Pelmear scale. "Tingling" and "numbness" are considered to accompany the blanching attacks, but there is no valid epidemiologic background for a quantified scaling of the occurrence of these symptoms in the various stages of the scale. Although, in an examination of 78 cases of the hand-arm vibration syndrome (11), a correlation has been demonstrated between the results of esthesiometer readings (two-point discrimination and depth sense) and Taylor-Pelmear staging, this correlation failed on an individual basis. There seems to be little reason in clinical work on individuals to include the $0_{\mathrm{N}}$ and $0_{\mathrm{T}}$ stages or to make references to numbness in stages $1-4$. On the contrary, there is much to gain from uncoupling the two types of symptoms - particularly if a separate scale of neurological symptoms can be constructed so as to make it amenable to clinical and neurophysiological laboratory techniques. Indeed, promising progress has been made in developing methods for the assessment of tactile sensory impairment $(1,2,4)$, and the advancement is in line with these ambitions. Some new data have been collected concerning what tests of sensory modalities may be useful in group research (11). A purely neurological scale has been proposed (3) which makes combined use of results of neurophysiological tests and clinical observations.

\section{Disability criteria}

There are convincing reasons to delete the disability stages hitherto used in the Taylor-Pelmear scale. Leisure-time activities of, for instance, forestry workers, are often quite different from those of factory workers in an urban area. Furthermore, a subjective scale based on disability should not be used in conjunction with the vascular scale, as disability is often defined on the basis of nonmedical factors.

\section{Seasonal criteria}

Determination of staging without reference to seasonal criteria is consistent with the preceding considerations. The frequency of Raynaud attacks, within or outside work, in an occupational group commonly exposed to cold in outdoor work during wintertime obviously differs from that in a group of workers who perform their daily tasks indoors. Furthermore, variations in personal habits and idiosyncrasies concerning the need for and methods for protection against cold have a strong influence on the frequency of attacks.

Another circumstance worthy of being commented upon is the relationship between the various environ- mental factors to which the workers are exposed at work and the occurrence of white fingers. It seems to be a common experience that Raynaud's phenomenon does not typically occur during ongoing work with hand-held vibrating tools. The reason may be that heat produced in the muscles and other tissues of the hand by the manual work counteracts the influences of environmental cold that would otherwise trigger an attack (8).

\section{Objective testing}

The role of environmental cold in triggering Raynaud's phenomenon has been utilized in the laboratory for objective symptom testing. The methods currently employed - chiefly blood pressure measurements in the finger in connection with finger cooling and the occlusion of arterial blood flow ("critical opening pressure") (6) - are season-dependent despite the fact that the tests are performed under controlled laboratory conditions (5). Generally, the determination of critical opening pressure gives fewer falsely positive results (which means high specificity) in vibration-exposed than in unexposed persons. However, the proportion of falsely negative results is considerable, chiefly in summertime, for patients with a reliable history of white fingers (overall sensitivity about $75 \%)(5,13)$. For these reasons, it is not possible at present to base the staging on the results of currently employed laboratory tests.

\section{Distinction between stages}

Difficulty has been experienced by some researchers in differentiating the current stages from each other, in particular, stages 2 and 3 . It has been suggested that this problem may be overcome in a semi-quantitative way by counting the number of affected fingers, which is an obvious alternative method of improving the discriminative power of the stages. This method has been chosen for the revised version of the scale. For each hand a separate staging is made according to the basic scale and the number of affected fingers on each hand. The notation consists of a numeral indicating the stage, followed by an L (for left) or an R (for right), with the number of affected fingers within parentheses. Thus, in a case where Raynaud's phenomenon occurs as stage 2 in two fingers on the left hand and as stage 1 in one finger on the right hand, the notation would be " $2 \mathrm{~L}(2) / 1 \mathrm{R}(1)$ ". If Raynaud's phenomenon of stage 3 occurs in four fingers on the right hand, while the left hand is symptom-free, the result would be indicated by " $-/ 3 R(4)$ ".

Stage 4 has been defined for the severe but very rare cases with trophic skin changes in the finger extremities. The full text of the revised scale is given in table 1. 
The criteria descriptions indicate that Raynaud's phenomenon tends to occur more often with increasing severity. However, the distinction between stages should not be unduly based on attack frequency; as pointed out earlier, there may be differences between various populations in, for instance, climatic factors and activities outside work.

\section{Discussion}

\section{Preservation of scale usefulness}

The current Taylor-Pelmear scale is extensively used in the United Kingdom for medicolegal purposes. In some other countries it has been widely employed for symptom classification in clinical and research work. Therefore there is some justification not to change the scale unduly so as to avoid losing its continuity. We believe that with the proposed changes this aspect has been satisfactorily addressed.

There remains the question of reversibility of the symptoms, a topic often discussed during the past decade. There is recent evidence that, after a sufficient period of time, reversibility may be achieved for vibration-induced white finger, provided that the stimuli for vasoconstriction are eliminated from the total environment of the subject. If reversibility is proved, the sharp delineation between the current stages 2, 3 and 4 - although important for a correct staging in research - is not such an all-decisive issue as it was originally thought to be with respect to the usefulness of the scale for medicolegal purposes.

\section{Distinction between cold sensitivity and true Raynaud's phenomenon}

Care should be taken to distinguish between symptoms of peripheral cold sensitivity in a general sense and those pointing to true Raynaud's phenomenon. "Constitutional Raynaud", a tendency towards a feeling of cold and diffuse pallor (rather than localized blanching) in the fingers and toes, may simulate white fingers and give the false impression of an initial phase of Raynaud's phenomenon. Inclusion of a patient with such symptoms in stage 1 of the scale will be confounding and should be avoided. In a large proportion of such cases, no clear-cut white fingers will ever develop.

The correct inclusion of a subject in stage 1 requires strict adherence to the criteria of typical Raynaud's phenomenon (secondary Raynaud) for vibrationexposed persons. These criteria comprise attacks of well-demarcated, local blanching and accompanying numbness of the affected parts of the finger skin triggered by exposure to environmental cold (general cooling of the body often being the most effective), with a distribution over the hands and fingers that agrees well with the strongest vibration exposure. In contrast to the acquired disorder, most people who suffer from the idiopathic type of digital vasospasm
Table 1. The Stockholm Workshop scale for the classification of cold-induced Raynaud's phenomenon in the hand-arm vibration syndrome. ${ }^{a}$

\begin{tabular}{|c|c|c|}
\hline Stage & Grade & Description \\
\hline 0 & & No attacks \\
\hline 1 & Mild & $\begin{array}{l}\text { Occasional attacks affecting only } \\
\text { the tips of one or more fingers }\end{array}$ \\
\hline 2 & Moderate & $\begin{array}{l}\text { Occasional attacks affecting distal } \\
\text { and middle (rarely also proximal) } \\
\text { phalanges of one or more fingers }\end{array}$ \\
\hline 3 & Severe & $\begin{array}{l}\text { Frequent attacks affecting all } \\
\text { phalanges of most fingers }\end{array}$ \\
\hline 4 & Very severe & $\begin{array}{l}\text { As in stage } 3 \text {, with trophic skin } \\
\text { changes in the finger tips }\end{array}$ \\
\hline
\end{tabular}

a The staging is made separately for each hand. In the evaluation of the subject, the grade of the disorder is indicated by the stages of both hands and the number of affected fingers on each hand; example: " $2 L(2) / 1 R(1)$ ", "- $/ 3 R(4)$ ", etc.

(primary Raynaud or Raynaud's disease) are women of fertile ages (prevalence about $15 \%$, as compared with about $5 \%$ among men). In these groups the finger blanching tends to be diffuse, as well as symmetrical, with or without a clear demarcation from surrounding skin.

\section{Numerical scoring}

The scale proposed by Rigby \& Cornish (9), based on scores for the blanching of different phalanges, must be considered unsatisfactory in two respects. The main objection is that only the sum score of the two hands is taken into account. In the cases where blanching occurs in only one hand, this important information is obscured by such a scoring system. Second, the method does not differentiate between the thumb and the other digits despite the fact that only in a small minority of cases is the thumb involved in the attacks of white fingers. In a recent investigation on miners (7), staging according to digit scores could not discriminate between stages 2 and 3 of the Taylor-Pelmear scale. After a consideration of the credits and the aforementioned drawbacks, it has been concluded that no advantage over the Taylor-Pelmear criteria seems to be derived from a scoring system based on the number of affected phalanges.

Nevertheless, a scoring system is needed to improve the discriminatory power of the staging. A scoring of the number of affected fingers separately for each hand is therefore proposed to be used in parallel with the staging according to the basic scale.

\section{References}

1. Brammer AJ, Piercy JE, Auger PL. Assessment of impaired tactile sensation: A pilot study. Scand J Work Environ Health 13 (1987) 380-384.

2. Brammer AJ, Pyykkö I. Vibration-induced neuropathy: Detection by nerve conduction measurements. Scand J 
Work Environ Health 13 (1987) 317-322.

3. Brammer AJ, Taylor W, Lundborg G. Sensorineural stages of the hand-arm vibration syndrome. Scand J Work Environ Health 13 (1987) 279-283.

4. Brammer AJ, Taylor W, Piercy JE. Assessing the severity of the neurological component of the hand-arm vibration syndrome. Scand J Work Environ Health 12 (1986) 428-431.

5. Ekenvall L, Lindblad L-E. Vibration white finger and digital systolic pressure during cooling. $\mathrm{Br} \mathrm{J}$ Ind Med 43 (1986) 280-283.

6. Olsen N, Nielsen SL. Diagnosis of Raynaud's phenomenon in quarrymen's traumatic vasospastic disease. Scand J Work Environ Health 5 (1979) 249-256.

7. Pelmear PL, Roos J, Leong D, Wong L. Cold provocation test results from a 1985 survey of hard-rock miners in Ontario. Scand J Work Environ Health 13 (1987) $343-347$.

8. Pyykkö I. The prevalence and symptoms of traumatic vasospastic disease among lumberjacks in Finland: A field study. Work Environ Health 11 (1974) 118-131.

9. Rigby TA, Cornish D. "Vibration Syndrome Research Panel." Guest, Keen, and Nettlefolds Ltd (GKN Forgings Ltd) and Rolls-Royce, Derby (England) 1984.

10. Taylor W. Occupational disability arising from the Vibration Syndrome. In: Gemne G, Taylor W, ed. Handarm vibration syndrome and the central autonomic nervous system. J Low Freq Noise Vib 1 (1983): special issue, $1-5$.

11. Taylor W, Ogston SA, Brammer AJ. A clinical assessment of seventy-eight cases of hand-arm vibration syndrome. Scand J Work Environ Health 12 (1986) 265268.

12. Taylor W, Pelmear PL, ed. Vibration white finger in industry. Academic Press, London 1975, p xxi.

13. Thulesius O, Brubakk A, Berlin E. Response of digital blood pressure to cold provocation in cases with Raynaud phenomena. Angiology 32 (1981) 113-118. 